\title{
Mapping Crystallization Kinetics of Phase-Change Materials Over Large Temperature Ranges Using Complementary In Situ Microscopy Techniques
}

\author{
Victoriea L. Bird, ${ }^{1}$ Al J. Rise, ${ }^{1}$ Khim Karki, ${ }^{2}$ Daan Hein Alsem, ${ }^{2}$ Geoffrey H. Campbell, ${ }^{3}$ and Melissa K. \\ Santala ${ }^{1}$ \\ 1. Mechanical, Industrial \& Manufacturing Engineering, Oregon State University, Corvallis, OR, USA \\ 2. Hummingbird Scientific, Lacey, WA, USA \\ 3. Materials Science Division, Lawrence Livermore National Laboratory, Livermore, CA, USA
}

Chalcogenide-based phase change materials (PCMs) materials are alloys with distinct contrast in optical and electrical properties in the amorphous and crystalline phases that make them valuable for optical and resistivity-based solid-state memory devices [1]. It is possible to switch between the amorphous and crystalline phases in nanoseconds at high temperature, but the amorphous phases may also be stable against crystallization at device operating temperatures for years. Relative to other glass forming materials, PCMs may be considered marginal glass formers. The crystallization kinetics of PCMs are important as they impact recording speed and long-term stability, but even basic quantities, such as crystal growth rates as a function of temperature are difficult to measure experimentally. Growth rates may span $>9$ orders of magnitude between solid-state low temperature growth (that can affect long termstability) and the peak growth rate [2,3]. However, such measurements may lead to a fundamentally better understanding of the crystallization kinetics of these and other marginal glass formers.

Ag-In-Sb-Te alloys have been used for memory devices [1] and have been widely studied, including efforts to map the crystal growth rate over large temperature ranges [4]. We measured the crystallization kinetics of $30-\mathrm{nm}$ amorphous thin films with a nominal composition of $\mathrm{Ag}_{3} \mathrm{In}_{4} \mathrm{Sb}_{74} \mathrm{Te}_{17}$ using complementary in situ microscopy techniques in order to cover a broad temperature range. At low temperatures, growth was measured using optical microscopy. At intermediate temperatures, a TEM heating holder with a MEMS-based chip with biasing and heating configurations was used for heating in a JEOL JEM-2100 and images were recorded using Direct Electron DE-12 camera with acquisition rates of 20-30 fps (Figure 1). At high temperatures, growth rates were measured with dynamic transmission electron microscopy (DTEM), a photo-emission electron microscopy technique with nanosecond-scale time resolution. Crystallization was induced with a laser directed onto the specimen in the TEM column. Nine-frame movies of crystal growth were taken over a periods of a few microseconds, e.g. Figure 2. Using this variety of in situ imaging techniques, the crystallization kinetics of this Ag-In-Sb-Te alloy will be mapped more completely as a function of temperature. This methodology will lead to a more comprehensive understanding of the crystallization kinetics in technologically-important marginal glass formers $[3,5]$.

References:

[1] S. Raoux, Annual Review of Materials Rsearch 39 (2009), p. 25.

[2] M.K. Santala et al., Applied Physics Letters 102 (2013), p. 174105

[3] J. Orava and A. L. Greer, Journal of Chemical Physics 140 (2014), p. 214504.

[4] M. Salinga et al., Nature Communications 4 (2013), p. 1.

[5] This work was performed, in part, under the auspices of the U.S. Department of Energy, Office of

Basic Energy Sciences, Division of Materials Sciences and Engineering by Lawrence Livermore

National Laboratory under Contract DE-AC52-07NA27344. 

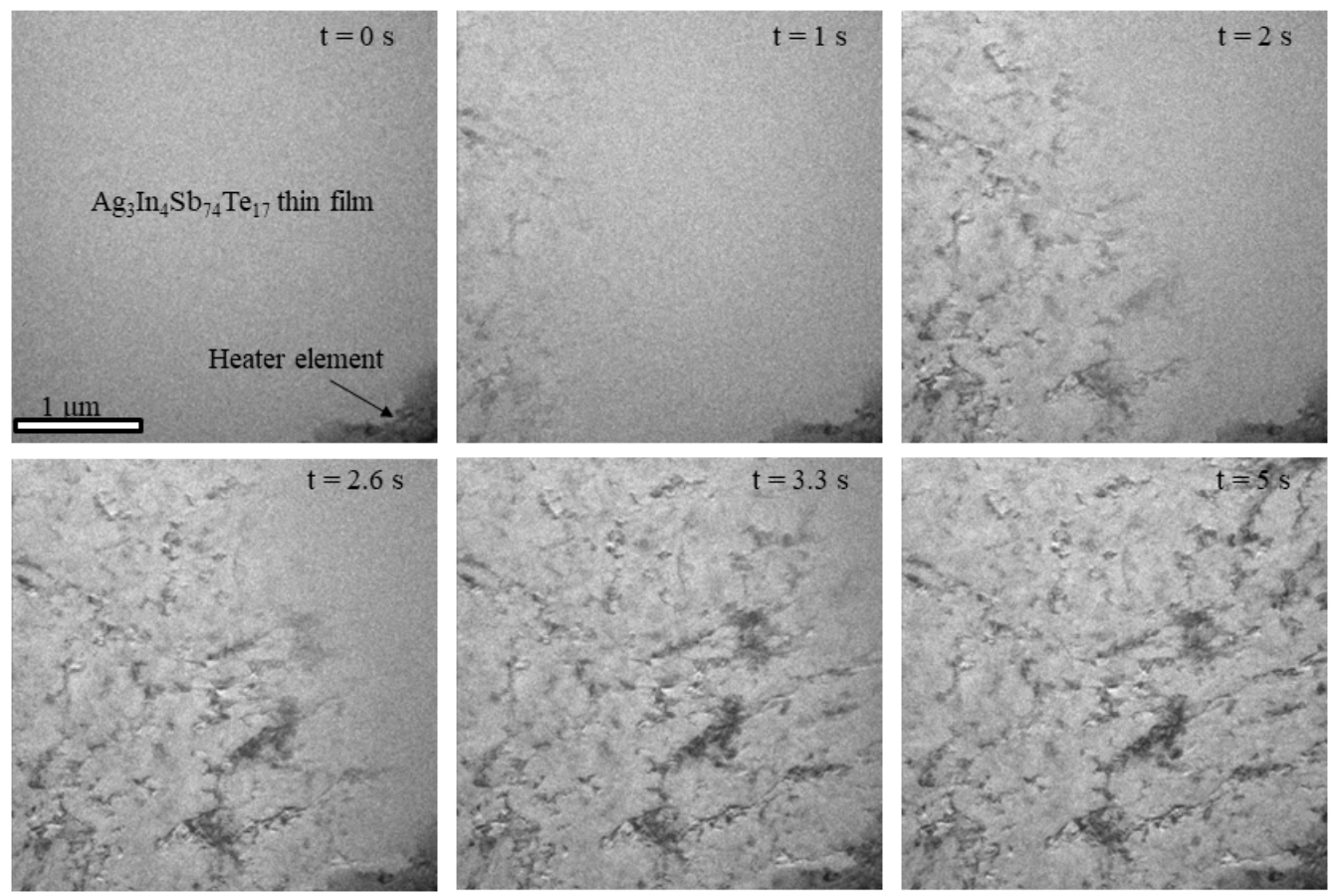

Figure 1. Bright field TEM images during amorphous-to-crystalline phase transformation in Ag-In-SbTe alloy thin film using a MEMS-based heater and direct electron detection camera. This method allows the growth to be tracked at temperature where crystal growth proceeds at $\sim 1 \mu \mathrm{m} / \mathrm{s}$.

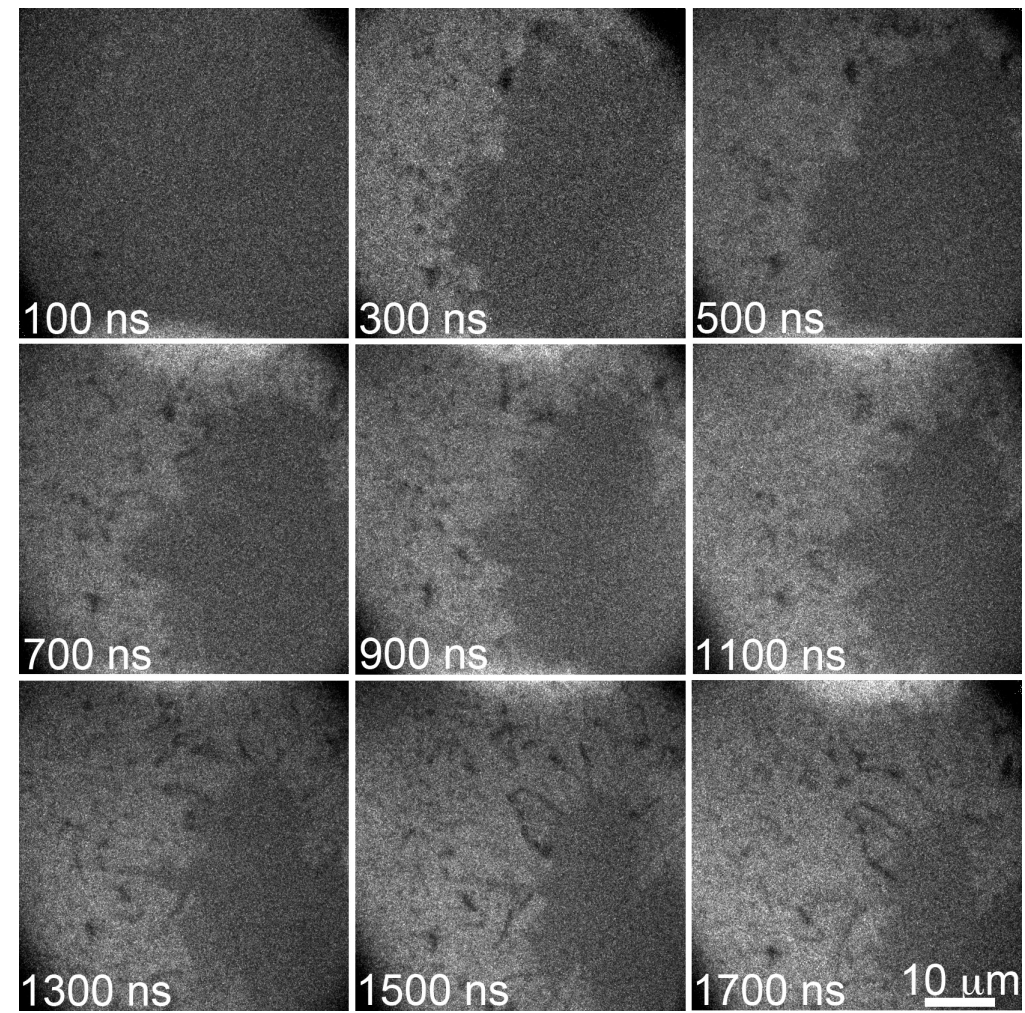

Figure 2. DTEM images formed by nine 50-ns electron pulses show the growth of crystalline grains in an amorphous Ag-In-Sb-Te thin film induced by laser heating. Times are relative to the specimen laser intensity with an uncertainty of $\pm 3 \mathrm{~ns}$. Growth rates exceeding $10 \mathrm{~m} / \mathrm{s}$ may be measured with DTEM. 